\title{
On the profile of the corona of two graphs
}

\author{
Yung-Ling Lai ${ }^{\text {a }}$, Gerard J. Chang ${ }^{\text {b,* }}$ \\ ${ }^{\text {a }}$ Graduate Institute of Computer Science and Information Engineering, National Chia-Yi University, Chiayi, Taiwan \\ b Department of Mathematics, National Taiwan University, Taipei 106, Taiwan
}

Received 3 November 2003; received in revised form 10 December 2003

Communicated by S. Albers

\begin{abstract}
The concept of profile, together with bandwidth, originates from handling sparse matrices in solving linear systems of equations. Given a graph $G$, the profile minimization problem is to find a one-to-one mapping $f: V(G) \rightarrow\{1,2, \ldots,|V(G)|\}$ such that $\sum_{v \in V(G)} \max _{x \in N[v]}(f(v)-f(x))$ is as small as possible, where $N[v]=\{v\} \cup\{x: x$ is adjacent to $v\}$. This paper studies the profile of the corona $G \wedge H$ of two graphs $G$ and $H$. In particular, bounds for the profile of the corona of two graphs are established. Also, exact values of the profiles of coronas $G \wedge H$ are obtained when $G$ has certain properties, including when $G$ is a caterpillar, a complete graph or a cycle.
\end{abstract}

(c) 2003 Elsevier B.V. All rights reserved.

Keywords: Profile; Corona; Numbering; Interval graph; Caterpillar; Complete graph; Cycle

\section{Introduction}

The concept of profile, together with bandwidth, originates from handling sparse matrices in solving linear systems of equations, see $[2,8,11,12,17]$ and their references. This problem can be re-formulated as the following problem in graphs.

A numbering (or labeling or layout) of a graph $G$ is a one-to-one mapping $f$ from $V(G)$ onto $\{1,2, \ldots$, $|V(G)|\}$. For a numbering $f$, the profile-width of a vertex $v$ is defined as

$w_{f}(v)=\max _{x \in N[v]}(f(v)-f(x))$,

\footnotetext{
* Corresponding author. Supported in part by the National Science Council under grant NSC92-2115-M002-015.

E-mail addresses: yllai@mail.ncyu.edu.tw (Y.-L. Lai), gjchang@math.ntu.edu.tw (G.J. Chang).
}

where $N[v]=\{x \in V(G): x=v$ or $x v \in E(G)\}$ is the closed neighborhood of $v$. The profile of a numbering $f$ of $G$ is

$P_{f}(G)=\sum_{v \in V(G)} w_{f}(v)$

and the profile (or skyline) of $G$ is

$P(G)=\min \left\{P_{f}(G): f\right.$ is a numbering of $\left.G\right\}$.

A numbering $f$ is called a profile numbering of $G$ if $P_{f}(G)=P(G)$.

The profile minimization problem has many equivalent definitions, such as interval graph completion $[1,20]$ and graph searching [5]. In this paper, we are more concerned about the interval graph completion. A graph $G=(V, E)$ is an interval graph if we can associate each vertex $v$ with a (closed) interval $I_{v}$ in the real line such that two different vertices $x$ and $y$ are 
adjacent if and only if $I_{x} \cap I_{y} \neq \emptyset$. The interval graph completion problem is for a graph $G$ finding a supergraph of $G$ (which is a graph $G^{\prime}$ with $V\left(G^{\prime}\right)=V(G)$ and $E(G) \subseteq E\left(G^{\prime}\right)$ ) of minimum size that is an interval graph.

Proposition 1 [1,20]. For any graph $G$ the profile of $G$ is equal to the smallest number of edges in an interval supergraph of $G$.

The interval graph completion problem is known to be NP-complete even for edge graphs (see [7]). On the other hand, there are linear-time algorithms for cographs [5,14] and an $\mathrm{O}\left(n^{1.722}\right)$-time algorithm for trees of $n$ vertices $[14,15]$. Because of its important applications, quite a few approximation algorithms have also been developed, see $[13,23,28-30]$. We say that the profile minimization problem is solved for a class of graphs if either a polynomial-time numbering algorithm which achieves the profile for each graph in the class is provided or a formula of the exact value is given for each graph in the class. The profile minimization problem is solved only for a few classes of graphs (most are graphs obtained from two graphs applying some graph operations), see $[9,16-18,20,21,24]$. Lai and Williams [19] provide a survey of recent works. The current paper is to study the profile of the corona of two graphs, which was introduced in [6].

Definition. Given graphs $G$ and $H$ with $n$ and $m$ vertices, respectively, the corona of $G$ with respect to $H$ is the graph $G \wedge H$ with vertex set $V(G \wedge H)$ $=V(G) \cup\left\{n\right.$ distinct copies of $V(H)$ denoted $V\left(H_{1}\right)$, $\left.V\left(H_{2}\right), \ldots, V\left(H_{n}\right)\right\}$ and edge set $E(G \wedge H)=E(G) \cup$ $\left\{n\right.$ distinct copies of $E(H)$ denoted $E\left(H_{1}\right), E\left(H_{2}\right)$, $\left.\ldots, E\left(H_{n}\right)\right\} \cup\left\{\left(u_{i}, v\right): u_{i} \in V(G), v \in V\left(H_{i}\right)\right\}$.

Fig. 1 shows $P_{3} \wedge P_{4}$.

Graph parameters such as bandwidth, edgesum, and domination number have been studied on coronas of graphs, see $[3,4,10,27,31]$. Also, the incidence

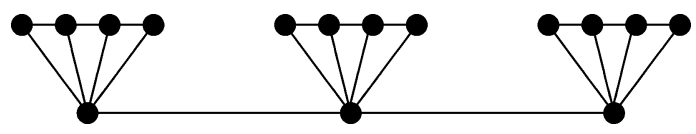

Fig. 1. $P_{3} \wedge P_{4}$. coloring of corona graphs has been studied by several researchers, see $[22,25,26]$. In this paper we establish bounds for the profile of the corona of two graphs. Exact values of the profiles of coronas $G \wedge H$ are determined when $G$ has certain properties, including when $G$ is a caterpillar, a complete graph or a cycle.

\section{Bounds for general graphs}

In this section we establish bounds for the profile of the corona of two general graphs. For convenience, we introduce the following terminology.

An interval-labeling of a graph $G$ (which is not necessary an interval graph) is a mapping $f$ on $V(G)$ that maps each vertex $v$ to a (closed) interval $f(v)$ such that $x y \in E(G)$ implies $f(x) \cap f(y) \neq \emptyset$. Let $I_{f}(G)$ denote the number of unordered pairs $\{f(x), f(y)\}$ with $x \neq y$ for which $f(x) \cap f(y) \neq \emptyset$; and $I(G)$ denoted the minimum value of $I_{f}(G)$, where $f$ is taken over all interval-labellings of $G$. An interval-labeling $f$ is called $I$-optimal if $I_{f}(G)=$ $I(G)$. According to Proposition $1, P(G)=I(G)$ for any graph $G$. We will use $P(G)$ and $I(G)$ interchangeably.

In the above definition, we may assume that $f$ is canonical (i.e., the $2|V(G)|$ endpoints of the intervals $f(v)$ are all distinct) as this does not affect the value of $I(G)$. In particular, each interval $f(v)$ is non-trivial (i.e., has a positive length) and the intersection of any two intersecting intervals $f(x)$ and $f(y)$ is also non-trivial. We assume that all interval-labellings are canonical throughout this paper.

Suppose $f$ is an interval-labeling of a graph $G$. For any vertex $v$, denoted by $M_{f}(v)$ the minimum number of intervals $f(x)$ intersecting a non-trivial interval $J_{v}$ with $J_{v} \cap f(v) \neq \emptyset$, where $x$ is taken over all vertices in $G$ and $J_{v}$ is taken over all possible intervals with $J_{v} \cap f(v) \neq \emptyset$. Notice that $M_{f}(v)$ is a finite number even if there are infinitely many possible $J_{v}$ as there are only finitely many $f(x)$. Denote by $M_{f}(G)$ the $\operatorname{sum} \sum_{v \in V} M_{f}(v)$, and $M(G)=\min \left\{M_{f}(G): f\right.$ is an interval-labeling of $G$ \}. An interval-labeling $f$ is called $M$-optimal if $M_{f}(G)=M(G)$.

In the above definition, we may assume that $J_{v}$ is a non-trivial subinterval of $f(v)$ and all $J_{v}$ s are pairwise disjoint, as this does not affect the value of $M(G)$.

We are now ready to establish a lower bound for the profile $P(G \wedge H)$ in terms of $P(G), P(H)$ 
and $M(G)$. We assume that $V(G)=\left\{u_{1}, u_{2}, \ldots, u_{n}\right\}$ and the copy of $H$ corresponding to $u_{i}$ is $H_{i}$ with vertex set $V\left(H_{i}\right)=\left\{v_{i 1}, v_{i 2}, \ldots, v_{i m}\right\}$ for $1 \leqslant i \leqslant n$.

Lemma 2. If $G$ and $H$ are two graphs of orders $n$ and $m$, respectively, then

$$
\begin{aligned}
& P(G \wedge H) \\
& =\min \left\{I_{g}(G)+n P(H)+M_{g}(G) m:\right. \\
& \quad g \text { is an interval-labeling of } G\} .
\end{aligned}
$$

Proof. Suppose $f^{*}$ is an $I$-optimal interval-labeling of $G \wedge H$. Let $g^{\prime}$ be the restriction of $f^{*}$ on $V(G)$ and $h_{i}^{\prime}$ the restriction of $f^{*}$ on $V\left(H_{i}\right)$ for $1 \leqslant i \leqslant n$. Then

$$
\begin{aligned}
P(G \wedge H) & =I_{f^{*}}(G \wedge H) \\
& \geqslant I_{g^{\prime}}(G)+\sum_{i=1}^{n} I_{h_{i}^{\prime}}\left(H_{i}\right)+\sum_{j=1}^{m} \sum_{i=1}^{n} b_{i j},
\end{aligned}
$$

where $b_{i j}$ is the number of intervals $g^{\prime}\left(u_{k}\right)$ s that intersect the interval $h_{i}^{\prime}\left(v_{i j}\right)$. Notice that the inequality may possibly be strict if $f^{*}\left(v_{i j}\right)$ intersects $f^{*}\left(v_{i^{\prime} j^{\prime}}\right)$ for some $i \neq i^{\prime}$. As $h_{i}^{\prime}\left(v_{i j}\right) \cap g^{\prime}\left(u_{i}\right) \neq \emptyset$, we may choose $h_{i}^{\prime}\left(v_{i j}\right)$ as $J_{u_{i}}$ in the definition of $M_{g^{\prime}}$ to get $b_{i j} \geqslant M_{g^{\prime}}\left(u_{i}\right)$. Then

$$
\begin{aligned}
P(G \wedge H) & \geqslant I_{g^{\prime}}(G)+\sum_{i=1}^{n} P\left(H_{i}\right)+\sum_{j=1}^{m} \sum_{i=1}^{n} M_{g^{\prime}}\left(u_{i}\right) \\
& \geqslant \text { right-hand side of Eq. (1). }
\end{aligned}
$$

On the other hand, suppose $g^{*}$ is an intervallabeling of $G$ that attains the minimum in the righthand side of Eq. (1). For each $u_{i}$ in $V(G)$ there is an interval $J_{u_{i}}$ with $J_{u_{i}} \cap g^{*}\left(u_{i}\right) \neq \varnothing$ that intersects $M_{g^{*}}\left(u_{i}\right)$ intervals $g^{*}\left(u_{k}\right)$ s. Without loss of generality, we may assume that each $J_{u_{i}}$ is a non-trivial subinterval of $g^{*}\left(u_{i}\right)$ and all $J_{u_{i}}$ s are pairwise disjoint. For each $i$ we may choose an $I$-optimal interval-labeling $h_{i}^{*}$ of $H_{i}$ such that each $h_{i}^{*}\left(v_{i j}\right)$ is a subinterval of $J_{u_{i}}$. Define the mapping $f^{\prime}$ on $V(G \wedge H)$ by

$f^{\prime}(x)= \begin{cases}g^{*}(x), & \text { if } x \in V(G) ; \\ h_{i}^{*}(x), & \text { if } x \in V\left(H_{i}\right) .\end{cases}$

It is clear that $f^{\prime}$ is an interval-labeling of $G \wedge H$ with

$$
\begin{aligned}
P(G \wedge H) & \leqslant I_{f^{\prime}}(G \wedge H) \\
& =I_{g^{*}}(G)+\sum_{i=1}^{n} I_{h_{i}^{*}}\left(H_{i}\right)+\sum_{j=1}^{m} \sum_{i=1}^{n} b_{i j},
\end{aligned}
$$

where $b_{i j}$ is the number of intervals $g^{*}\left(u_{k}\right) \mathrm{s}$ that intersects the interval $h_{i}^{*}\left(v_{i j}\right)$. Then $b_{i j} \leqslant M_{g^{*}}\left(u_{i}\right)$ and so

$$
\begin{aligned}
P(G \wedge H) & \leqslant I_{g^{*}}(G)+\sum_{i=1}^{n} P\left(H_{i}\right)+\sum_{j=1}^{m} \sum_{i=1}^{n} M_{g^{*}}\left(u_{i}\right) \\
& =I_{g^{*}}(G)+n P(H)+M_{g^{*}}(G) m .
\end{aligned}
$$

Both inequalities prove the theorem.

Consequently, we have the following lower bound for the profile of the corona of two graphs.

Theorem 3. If $G$ and $H$ are two graphs of orders $n$ and $m$, respectively, then

$$
P(G \wedge H) \geqslant P(G)+n P(H)+M(G) m .
$$

Proof. The theorem follows from Lemma 2 and the fact that $I_{g}(G) \geqslant P(G)$ and $M_{g}(G) \geqslant M(G)$ for any interval-labeling $g$ of $G$.

The minimization in Eq. (1) depends not only on $G$ but also on the value $m$. It is also the case that the minimization is attained for the "mixed" value of both $I_{g}$ and $M_{g}$, rather than individual $I_{g}$ and $M_{g}$. However, if there is a $g^{*}$ that attains the minimum individually for $I_{g}$ and $M_{g}$ at the same time, then the lower bound in Theorem 3 is in fact the exact value.

Theorem 4. If $G$ has an interval-labeling $g^{*}$ that is both I-optimal and M-optimal, then

$P(G \wedge H)=P(G)+n P(H)+M(G) m$.

Proof. The theorem follows from Lemma 2 and the fact that $g^{*}$ attains the minimum of the right-hand side of Eq. (1).

Next, we establish an upper bound for the profile of the corona of two graphs in terms of their profiles.

Theorem 5. If $G$ and $H$ are two graphs of orders $n$ and $m$, respectively, then

$P(G \wedge H) \leqslant(m+1) P(G)+n P(H)+n m$. 
Proof. Let $g$ be a profile numbering of $G$ and $h$ be a profile numbering of $H$. Define a numbering $f$ of $G \wedge H$ as follows:

$$
f(x)=\left\{\begin{array}{l}
(m+1) g(x), \quad \text { if } x \in V(G) ; \\
(m+1)(g(y)-1)+h(x), \\
\quad \text { if } x \in V(H) \text { and }(x, y) \in E(G \wedge H) .
\end{array}\right.
$$

Then $P(G \wedge H) \leqslant P_{f}(G \wedge H) \leqslant(m+1) P(G)+$ $n P(H)+n m$.

The upper bound in Theorem 5 is tight as illustrated by $E_{n} \wedge H$, where $E_{n}$ is the complement of the complete graph $K_{n}$. Note that $E_{n} \wedge H$ is $n$ separate copies of $K_{1} \wedge H$ whose profile $P\left(K_{1} \wedge H\right)=P(H)+$ $m$, and then $P\left(E_{n} \wedge H\right)=n(P(H)+m)=(m+$ 1) $P\left(E_{n}\right)+n P(H)+n m$.

\section{Exact values}

This section establishes exact values of the profiles of coronas $G \wedge H$ by means of Theorem 4. These include when $G$ is a caterpillar, a complete graph or a cycle, for which the values of $P(G)$ and $M(G)$ are also determined.

We first consider the case when $G$ is a caterpillar. A caterpillar is a tree from whom the removing of all leaves results a path (possibly empty). More precisely, suppose $n=r+\sum_{1<i<r} s_{i}$, where $r \geqslant 2$ and each $s_{i} \geqslant 0$. A caterpillar with the parameters $\left(n ; r ; s_{2}, s_{3}, \ldots, s_{r-1}\right)$ is the tree $T$ with

vertex set $V(T)=\left\{x_{1}, x_{2}, \ldots, x_{r}\right\}$

$$
\cup \bigcup_{1<i<r}\left\{y_{i 1}, y_{i 2}, \ldots, y_{i s_{i}}\right\}
$$

and

$$
\text { edge set } \begin{aligned}
E(T)= & \left\{x_{1} x_{2}, x_{2} x_{3}, \ldots, x_{r-1} x_{r}\right\} \\
& \cup \bigcup_{1<i<r}\left\{x_{i} y_{i 1}, x_{i} y_{i 2}, \ldots, x_{i} y_{i s_{i}}\right\} .
\end{aligned}
$$

Theorem 6. If $T$ is a caterpillar with parameters $\left(n ; r ; s_{2}, s_{3}, \ldots, s_{r-1}\right)$ and $H$ is a graph of $m$ vertices, then $P(T)=n-1$ and $M(T)=2 n-r$ and

$$
\begin{aligned}
P(T \wedge H) & =P(T)+n P(H)+M(T) m \\
& =n-1+n P(H)+(2 n-r) m .
\end{aligned}
$$

Proof. First, it is clear that $P(T) \geqslant|E(T)|=n-1$.

Next, suppose $g$ is an $M$-optimal interval-labeling of $T$. Suppose $u$ is the vertex for which $g(u)$ has the smallest left endpoint, and $v$ is the vertex other than $u$ for which $g(v)$ has the largest right endpoint. Let $Q$ be the $u-v$ path in $T$, and assume it has $q$ vertices. Then, $\bigcup_{w \in V(Q)} g(w)$ is an interval in the real line that includes $g(z)$ for any vertex $z \in V(T)$. Consequently, $M_{g}(w) \geqslant 1$ for each $w \in V(Q)$ and $M_{g}(z) \geqslant 2$ for each $z \in V(T)-V(Q)$. As $q \leqslant r$, we have $M(T) \geqslant$ $q+2(n-q)=2 n-q \geqslant 2 n-r$.

On the other hand, define an interval-labeling $g^{*}$ by

$g^{*}(z)=\left\{\begin{array}{c}{[4 i, 4 i+5], \quad \text { if } z=x_{i}, 1 \leqslant i \leqslant r ;} \\ {\left[4 i+2+\frac{2 j}{2\left(s_{i}+1\right)}, 4 i+2+\frac{2 j+1}{2\left(s_{i}+1\right)}\right],} \\ \quad \text { if } z=y_{i j}, 1<i<r, 1 \leqslant j \leqslant s_{i} .\end{array}\right.$

It is clear that $g^{*}$ is an interval-labeling of $T$ with $I_{g^{*}}(T)=n-1$ and $M_{g^{*}}(T)=2 n-r$. Consequently, $g^{*}$ is both $I$-optimal and $M$-optimal, and $P(T)=$ $n-1$ and $M(T)=2 n-r$. The theorem then follows from Theorem 4.

We next consider the case when $G$ is the complete graph $K_{n}$.

Theorem 7. If $K_{n}$ is a complete graph of $n$ vertices and $H$ is a graph of $m$ vertices, then $P\left(K_{n}\right)=$ $n(n-1) / 2$ and $M\left(K_{n}\right)=\left\lfloor(n+1)^{2} / 4\right\rfloor$ and

$$
\begin{aligned}
P\left(K_{n} \wedge H\right)= & P\left(K_{n}\right)+n P(H)+M\left(K_{n}\right) m \\
= & n(n-1) / 2+n P(H) \\
& +\left\lfloor(n+1)^{2} / 4\right\rfloor m .
\end{aligned}
$$

Proof. First, it is clear that $P\left(K_{n}\right) \geqslant\left|E\left(K_{n}\right)\right|=$ $n(n-1) / 2$.

Next, suppose $g$ is an $M$-optimal interval-labeling of $K_{n}$. We recursively define $u_{1}, u_{2}, \ldots, u_{\lceil n / 2\rceil}$ and $v_{1}, v_{2}, \ldots, v_{\lfloor n / 2\rfloor}$ as follows. Having defined $u_{j}$ and $v_{j}$ for all $j<i$, let $u_{i}$ be the vertex (other than the above vertices) for which $g\left(u_{i}\right)$ has the smallest left endpoint, and $v_{i}$ is the vertex (other than the above vertices and $u_{i}$ ) for which $g\left(v_{i}\right)$ has the largest right endpoint. Repeat this process until all vertices of $K_{n}$ are scanned. It is the clear that for each $1 \leqslant i \leqslant$ $\lfloor n / 2\rfloor$ we have that $g\left(u_{i}\right) \cup g\left(v_{i}\right)$ is an interval in the real line that includes $g\left(u_{j}\right)$ and $g\left(v_{j}\right)$ for all $j \geqslant i$. Consequently, $M_{g}\left(u_{i}\right) \geqslant i$ for $1 \leqslant i \leqslant\lceil n / 2\rceil$ and 
$M_{g}\left(v_{i}\right) \geqslant i$ for $1 \leqslant i \leqslant\lfloor n / 2\rfloor$. Then $M_{g}\left(K_{n}\right) \geqslant 1+$ $2+\cdots+\lceil n / 2\rceil+1+2+\cdots+\lfloor n / 2\rfloor=\left\lfloor(n+1)^{2} / 4\right\rfloor$.

On the other hand, suppose $V\left(K_{n}\right)=\left\{x_{1}, x_{2}\right.$, $\left.\ldots, x_{n}\right\}$. Define $g^{*}$ by $g^{*}\left(x_{i}\right)=[i, i+n]$ for $1 \leqslant i \leqslant n$. It is clear that $g^{*}$ is an interval-labeling of $K_{n}$ with $I_{g^{*}}\left(K_{n}\right)=n(n-1) / 2$ and $M_{g^{*}}\left(K_{n}\right)=\left\lfloor(n+1)^{2} / 4\right\rfloor$. Consequently, $g^{*}$ is both $I$-optimal and $M$-optimal, and $P\left(K_{n}\right)=n(n-1) / 2$ and $M\left(K_{n}\right)=\left\lfloor(n+1)^{2} / 4\right\rfloor$. The theorem then follows from Theorem 4.

Finally, we consider the case when $G$ is the cycle $C_{n}$.

Theorem 8. If $C_{n}$ is a cycle of $n$ vertices and $H$ is a graph of $m$ vertices, then $P\left(C_{n}\right)=2 n-3$ and $M\left(C_{n}\right)=2 n-2$ and

$$
\begin{aligned}
P\left(C_{n} \wedge H\right) & =P\left(C_{n}\right)+n P(H)+M\left(C_{n}\right) m \\
& =2 n-3+n P(H)+(2 n-2) m .
\end{aligned}
$$

Proof. We first prove that $P\left(C_{n}\right) \geqslant 2 n-3$ by induction on $n$. The case of $n=3$ is clear as $P\left(C_{3}\right) \geqslant$ $\left|E\left(C_{3}\right)\right|=3$. Suppose $P\left(C_{n}\right) \geqslant 2 n-3$ for $n \geqslant 3$. Choose an $I$-optimal interval-labeling $g$ of $C_{n+1}$. Let $x$ be the vertex whose $g(x)$ has a smallest right endpoint. Then its two neighbors $y$ and $z$ both contain this right endpoint. Consequence $g(y) \cap g(z) \neq \emptyset$. Hence the restriction $g^{\prime}$ of $g$ on $V\left(C_{n+1}\right)-\{x\}$ is an interval-labeling of $C_{n}$. Then $P\left(C_{n+1}\right)=I_{g}\left(C_{n+1}\right) \geqslant$ $2+I_{g^{\prime}}\left(C_{n}\right) \geqslant 2+P\left(C_{n}\right) \geqslant 2+2 n-3=2(n+1)-3$. Therefore, $P\left(C_{n}\right) \geqslant 2 n-3$ for all $n \geqslant 3$.

Next, suppose $g$ is an $M$-optimal interval-labeling of $C_{n}$. Let $u$ be the vertex for which $g(u)$ has the smallest left endpoint, and $v$ be the vertex other than $u$ for which $g(v)$ has the largest right endpoint. Then $C_{n}$ is the union of two internally vertex-disjoint $u-v$ paths $Q_{1}$ and $Q_{2}$. It is the case that $g(x) \subseteq \bigcup_{y \in V\left(Q_{3-i}\right)} g(y)$ for each vertex $x \in V\left(Q_{i}\right), i=1,2$. Consequently, $M_{g}(x) \geqslant 2$ for all vertices in $C_{n}$ except $M_{g}(u) \geqslant 1$ and $M_{g}(v) \geqslant 1$. Then $M_{g}\left(C_{n}\right) \geqslant 2(n-2)+1+1=$ $2 n-2$.

On the other hand, suppose $V\left(C_{n}\right)=\left\{x_{1}, x_{2}\right.$, $\left.\ldots, x_{n}\right\}$. Define $g^{*}$ by $g^{*}\left(x_{i}\right)=[2 i, 2 i+3]$ for $1 \leqslant$ $i \leqslant n-1$ and $g^{*}\left(x_{n}\right)=[3,2 n+2]$. It is clear that $g^{*}$ is an interval-labeling of $C_{n}$ with $I_{g^{*}}\left(C_{n}\right)=2 n-3$ and $M_{g^{*}}\left(C_{n}\right)=2 n-2$. Consequently, $g^{*}$ is both $I$-optimal and $M$-optimal, and $P\left(C_{n}\right)=2 n-3$ and
$M\left(C_{n}\right)=2 n-2$. The theorem then follows from Theorem 4.

\section{Acknowledgements}

The authors thank the referees for many constructive suggestions.

\section{References}

[1] A. Billionnet, On interval graphs and matrix profiles, RAIRO Rech. Opér. 20 (1986) 245-256.

[2] P.Z. Chinn, J. Chvatalova, A.K. Dewdney, N.E. Gibbs, The bandwidth problem for graphs and matrices-a survey, J. Graph Theory 6 (1982) 223-254.

[3] P.Z. Chinn, Y. Lin, J. Yuan, The bandwidth of the corona of two graphs, Congr. Numer. 91 (1992) 141-152.

[4] J.F. Fink, M.S. Jacobson, L.F. Kinch, J. Roberts, On graphs having domination number half their order, Period. Math. Hungar. 16 (1985) 287-293.

[5] F.V. Fomin, P.A. Golovach, Graph searching and interval completion, SIAM J. Discrete Math. 13 (2000) 454-464.

[6] R. Frucht, F. Harary, On the coronas of two graphs, Aequationes Math. 4 (1970) 322-324.

[7] M.R. Garey, D.S. Johnson, Computers and Intractability: A Guide to the Theory of NP-Completeness, Freeman, San Francisco, CA, 1979.

[8] N.E. Gibbs, W.G. Poole Jr., P.K. Stockmeyer, An algorithm for reducing the bandwidth and profile of a sparse matrix, SIAM J. Numer. Anal. 13 (1976) 235-251.

[9] Y. Guan, K. Williams, Profile minimization problem on triangulated triangles, Comput. Sci. Dept. Tech. Report, TR/98-02, Western Michigan University, Kalamazoo, MI, 1998.

[10] T.W. Haynes, S.T. Hedetniemi, P.J. Slater, Fundamentals of Domination in Graphs, Marcel Dekker, New York, 1998.

[11] J. Jeffs, Effects of a local change on the bandwidth of a graph, Congr. Numer. 89 (1992) 45-53.

[12] I.P. King, An automatic reordering scheme for simultaneous equations derived from network systems, Internat. J. Numer. Methods Engrg. 2 (1970) 523-533.

[13] B.U. Koo, B.C. Lee, An efficient profile reduction algorithm based on the frontal ordering scheme and the graph theory, Comput. Structures 44 (6) (1992) 1339-1347.

[14] D. Kuo, The profile minimization problem in graphs, Master Thesis, Dept. Applied Math., National Chiao Tung Univ., Hsinchu, Taiwan, June 1991.

[15] D. Kuo, G.J. Chang, The profile minimization problem in trees, SIAM J. Comput. 23 (1) (1994) 71-81.

[16] D. Kuo, J.-H. Yan, The profile on $n$-cubes, Preprint, 2000.

[17] Y.-L. Lai, Bandwidth, Edgesum and profile of graphs, Ph.D. Thesis, Department of Computer Science, Western Michigan University, Kalamazoo, MI, 1997.

[18] Y.-L. Lai, Exact profile values of some graph compositions, Taiwanese J. Math. 6 (1) (2002) 127-134. 
[19] Y.-L. Lai, K. Williams, A survey of solved problems and applications on bandwidth, edgesum and profile of graphs, J. Graph Theory 31 (1999) 75-94.

[20] Y. Lin, J. Yuan, Profile minimization problem for matrices and graphs, Acta Math. Appl. Sinica, English-Series, Yingyong Shuxue-Xuebas 10 (1) (1994) 107-112.

[21] Y. Lin, J. Yuan, Minimum profile of grid networks, Systems Sci. Math. Sci. 7 (1) (1994) 56-66.

[22] X.K. Liu, Y. Li, Yan The incidence coloring of corona graphs, J. Xuzhou Norm. Univ. Nat. Sci. Ed. 19 (2) (2001) 16-18.

[23] J.C. Luo, Algorithms for reducing the bandwidth and profile of a sparse matrix, Comput. Structures 44 (3) (1992) 535-548.

[24] J. Mai, Profiles of some condensable graphs, J. System Sci. Math. Sci. 16 (1996) 141-148.
[25] W. Ning, L.Z. Zhang, The incidence coloring of corona graphs, J. Lanzhou Univ. Nat. Sci. 37 (3) (2001) 10-13.

[26] W. Ning, W. Zhang, F. Liu, The incidence coloring of corona graphs, J. Inn. Mong. Norm. Univ. 29 (2) (2000) 93-96.

[27] C. Payan, N.H. Xuong, Domination-balanced graphs, J. Graph Theory 6 (1982) 23-32.

[28] W.F. Smyth, Algorithms for the reduction of matrix bandwidth and profile, J. Comput. Appl. Math. 12\&13 (1985) 551-561.

[29] R.A. Snay, Reducing the profile of sparse symmetric matrices, Bull. Geod. 50 (1976) 341-352.

[30] M. Wiegers, B. Monien, Bandwidth and profile minimization, Lecture Notes in Comput. Sci. 344 (1988) 378-392.

[31] K. Williams, On the minimum sum of the corona of two graphs, Congr. Numer. 94 (1993) 43-49. 